\title{
A NOTE ON WEAK CONVERGENCE OF SINGULAR INTEGRALS IN METRIC SPACES
}

\author{
VASILIS CHOUSIONIS AND MARIUSZ URBAŃSKI
}

\begin{abstract}
We prove that in any metric space $(X, d)$ the singular integral operators$$
T_{\mu, \varepsilon}^{k}(f)(x)=\int_{X \backslash B(x, \varepsilon)} k(x, y) f(y) d \mu(y) .
$$

converge weakly in some dense subspaces of $L^{2}(\mu)$ under minimal regularity assumptions for the measures and the kernels.
\end{abstract}

\section{INTRODUCTION}

A Radon measure on a metric space $(X, d)$ has $s$-growth if there exists some constant $c_{\mu}$ such that $\mu(B(x, r)) \leq c_{\mu} r^{s}$ for all $x \in X, r>0$.

We say that $k(\cdot, \cdot): X \times X \backslash\{(x, y) \in X \times X: x=y\} \rightarrow \mathbb{R}$ is an $s$-dimensional kernel if there exists a constant $c>0$ such that for all $x, y \in X, x \neq y$ :

$$
|k(x, y)| \leq c d(x, y)^{-s} .
$$

The kernel $k$ is antisymmetric if $k(x, y)=-k(y, x)$ for all distinct $x, y \in X$.

Given a positive Radon measure $\nu$ on $X$ and an $s$-dimensional kernel $k$, we define

$$
T^{k} \nu(x):=\int k(x, y) d \nu(y), \quad x \in X \backslash \operatorname{spt} \nu .
$$

This integral may not converge when $x \in \operatorname{spt} \nu$. For this reason, we consider the following $\varepsilon$-truncated operators $T_{\varepsilon}^{k}, \varepsilon>0$ :

$$
T_{\varepsilon}^{k} \nu(x):=\int_{d(x, y)>\varepsilon} k(x, y) d \nu(y), \quad x \in X .
$$

Given a fixed positive Radon measure $\mu$ on $X$ and $f \in L_{\text {loc }}^{1}(\mu)$, we write

$$
T_{\mu}^{k} f(x):=T^{k}(f \mu)(x), \quad x \in X \backslash \operatorname{spt}(f \mu),
$$

and

$$
T_{\mu, \varepsilon}^{k} f(x):=T_{\varepsilon}^{k}(f \mu)(x) .
$$

Concerning the limit properties of the operators $T_{\mu, \varepsilon}^{k}$ one can ask if the limit, the so called principal value of $T$,

$$
\lim _{\varepsilon \rightarrow 0} T_{\mu, \varepsilon}^{k}(f)(x),
$$

2010 Mathematics Subject Classification. Primary 32A55, 30L99.

Key words and phrases. Singular integrals, metric spaces. 
exists $\mu$ almost everywhere. When $\mu$ is the Lebesgue measure in $\mathbb{R}^{d}$, and $k$ is a standard Calderón-Zygmund kernel, due to cancellations and the denseness of smooth functions in $L^{1}$, the principal values exist almost everywhere for $L^{1}$-functions. For more general measures, the question is more complicated. Let $n$ be an integer, $0<n<d$, and consider the coordinate Riesz kernels

$$
R_{i}^{n}(x)=\frac{x_{i}}{|x|^{n+1}} \text { for } i=1, \ldots, d .
$$

Tolsa proved in [T] that if $E \subset \mathbb{R}^{d}$ has finite $n$-dimensional Hausdorff measure $\mathcal{H}^{n}$ the principal values

$$
\lim _{\varepsilon \rightarrow 0} \int_{E \backslash B(x, \varepsilon)} \frac{x_{i}-y_{i}}{|x-y|^{m+1}} d \mathcal{H}^{n}(y)
$$

exist $\mathcal{H}^{n}$ almost everywhere in $E$ if and only if the set $E$ is $n$-rectifiable i.e. if there exist $n$-dimensional Lipschitz surfaces $M_{i}, i \in \mathbb{N}$, such that

$$
\mathcal{H}^{n}\left(E \backslash \cup_{i=1}^{\infty} M_{i}\right)=0 .
$$

Mattila and Preiss had obtained the same result earlier, in [MP] under some stronger assumptions for the set $E$. It becomes obvious that the existence of principal values is deeply related to the geometry of the set $E$.

Assuming $L^{2}(\mu)$-boundedness for the operators $T_{\mu}^{k}$ one could have expected that more could be deduced about the structure of $\mu$ and the existence of principal values, but this is a hard and, in a large extent, open problem. Dating from 1991 the David-Semmes conjecture, see [DS], asks if the $L^{2}(\mu)$-boundedness of the operators associated with the $n$-dimensional Riesz kernels suffices to imply $n$-uniform rectifiabilty, which can be thought as a quantitative version of rectifiability. In the very recent deep work [NToV], Nazarov, Tolsa and Volberg resolved the conjecture in the codimension 1 case, that is for $n=d-1$. Mattila, Melnikov and Verdera in [MMV], using a special symmetrization property of the Cauchy kernel, had earlier proved the conjecture in the case of 1-dimensional Riesz kernels. For all other dimensions and for other kernels few things are known. In fact, there are several examples of kernels whose boundedness does not imply rectifiability, see $[\mathrm{C}],[\mathrm{D}]$ and $[\mathrm{H}]$. For some recent positive results involving other kernels see [CMPT].

Let $\mu$ be a finite Radon measure and let $k$ be an antisymmetric kernel in a complete metric space $(X, d)$ where the Vitali covering theorem holds for $\mu$ and the family of closed balls defined by $d$. Mattila and Verdera in [MV] showed that in this case the $L^{2}(\mu)$-boundedness of the operators $T_{\mu, \varepsilon}^{k}$ forces them to converge weakly in $L^{2}(\mu)$. This means that there exists a bounded linear operator $T_{\mu}^{k}: L^{2}(\mu) \rightarrow L^{2}(\mu)$ such that for all $f, g \in L^{2}(\mu)$,

$$
\lim _{\varepsilon \rightarrow 0} \int T_{\mu, \varepsilon}^{k}(f)(x) g(x) d \mu(x)=\int T_{\mu}^{k}(f)(x) g(x) d \mu(x) .
$$

Furthermore notions of weak convergence have been recently used by Nazarov, Tolsa and Volberg in [NToV]. 
Motivated by these developments it is natural to ask if limits of this type might exist if we remove the very strong $L^{2}$-boundedness assumption. We prove that the operators $T_{\mu, \varepsilon}^{k}$ converge weakly in dense subspaces of $L^{2}(\mu)$ under minimal assumptions for the measures and the kernels in general metric spaces. Denote by $\mathcal{X}_{B}$ the space of all finite linear combinations of characteristic functions of balls in $X$,

$$
\mathcal{X}_{B}=\left\{\sum_{i=1}^{n} a_{i} \chi_{B\left(z_{i}, r_{i}\right)}: n \in \mathbb{N}, a_{i} \in \mathbb{R}, z_{i} \in X, r_{i}>0\right\} .
$$

Whenever Vitali's covering theorem holds for the closed balls in $(X, d)$ the space $\mathcal{X}_{B}$ is dense in $L^{2}(\mu)$. When $X=\mathbb{R}^{d}$ Vitali's covering theorem holds for any Radon measure $\mu$ and the closed balls defined by various metrics (including the standard $d_{p}$ metrics for $\left.1 \leq p \leq \infty\right)$ as a consequence of Besicovitch's covering theorem, see [M, Theorem 2.8]. Furthermore Vitali's covering theorem holds for any metric space $(X, d)$ whenever $\mu$ is doubling, that is when there exists some constant $C$ such that for all balls $B, \mu(2 B) \leq C \mu(B)$, see [F, Section 2.8].

Theorem 1.1. Let $\mu$ be a finite Radon measure with s-growth and $k$ an antisymmetric s-dimensional kernel on a metric space $(X, d)$. If the Vitali Covering theorem holds for the closed balls in $(X, d)$ then there exists subsets $\mathcal{X}_{B}^{\prime} \subset \mathcal{X}_{B}$ which are dense in $L^{2}(\mu)$ and the weak limits

$$
\lim _{\varepsilon \rightarrow 0} \int T_{\mu, \varepsilon}^{k} f(x) g(x) d \mu(x)
$$

exist for all $f, g \in \mathcal{X}_{B}^{\prime}$.

Until now Theorem 1.1 was only known for measures with $(d-1)$-growth in $\mathbb{R}^{d}$ under some smoothness assumptions for the kernels, see [CM]. We thus extend the result from [CM] to measures with $s$-growth for arbitrary $s$ in metric spaces where Vitali's covering theorem holds for the family of closed balls without requiring any smoothness for the kernels. Our proof follows a completely different strategy using an "exponential growth" lemma for probability measures on intervals and is self contained (unlike the proof from [CM] which depends on several $L^{2}(\nu)$ to $L^{2}(\mu)$ boundedness results for separated measures $\nu$ and $\mu$ ).

Recall that if $k$ is the $(d-1)$-dimensional Riesz kernel in $\mathbb{R}^{d}$ and $\mu$ has $(d-1)$ growth and is $(d-1)$ purely unrectifiable, that is $\mu(E)=0$ for all $(d-1)$-rectifiable sets $E$, the principal values diverge $\mu$ almost everywhere and the weak convergence in $L^{2}(\mu)$ fails. On the other hand it is of interest that weak convergence in the sense of Theorem 1.1 holds as it holds for any $s$-dimensional antisymmetric kernel and any finite measure with $s$-growth.

\section{Proof of Theorem 1.1}

We first prove the following lemma about exponential growth of probability measures on compact intervals. It is motivated by a similar result proved in [SUZ]. Here 
Leb stands for the Lebesgue measure on the real line and $|I|$ denotes the length of an interval $I \subset \mathbb{R}$.

Lemma 2.1. For every integer $\lambda>2$ the following holds. Let $\nu$ be a probability Borel measure on a compact interval $\Delta \subset \mathbb{R}$. Then for every interval $I \subset \Delta$ there exists a subset $I^{\prime}(\lambda) \subset I$ such that Leb $\left(I^{\prime}(\lambda)\right)>|I|\left(1-3\left(\lambda^{-1}+\lambda^{-2}+\ldots\right)\right)$ and for every $t \in I^{\prime}(\lambda)$,

$$
\nu\left(\left[t-\lambda^{3 n}, t+\lambda^{3 n}\right]\right)<\lambda^{-3 n}
$$

for all integers $n \geq 1$.

Proof. Let us partition the interval $I$ into $\lambda^{2}$ subintervals $J$ of length $|I| \lambda^{-2}$. Let $B_{1}$ be the family of all intervals $J$ from this partition for which $\nu(J)<\lambda^{-1}$. Obviously, there are at most $\lambda$ intervals in $B_{1}^{c}$. Thus

$$
\# B_{1}>\lambda^{2}-\lambda=\lambda^{2}\left(1-\frac{\lambda}{\lambda^{2}}\right)
$$

and

$$
\text { Leb }\left(\bigcup\left\{J: J \in B_{1}\right\}\right) \geq|I|\left(1-\frac{\lambda}{\lambda^{2}}\right)=|I|\left(1-\frac{1}{\lambda}\right) .
$$

Next, each interval in $B_{1}$ is divided into $\lambda^{2}$ subintervals with disjoint interiors and of length $|I| \lambda^{-4}$, and we remove those subintervals for which $\nu(J) \geq \lambda^{-2}$. Denoting by $B_{2}$ the family of remaining intervals, we see that

$$
\# B_{2} \geq\left(\lambda^{2}\right)^{2}\left(1-\frac{\lambda}{\lambda^{2}}\right)-\lambda^{2}=\left(\lambda^{2}\right)^{2}\left(1-\frac{1}{\lambda}-\frac{1}{\lambda^{2}}\right)
$$

and

$$
\operatorname{Leb}\left(\bigcup\left\{J: J \in B_{2}\right\}\right) \geq|I|\left(1-\frac{1}{\lambda}-\frac{1}{\lambda^{2}}\right) .
$$

Proceeding inductively, we partition the interval $I$ into disjoint intervals of length $|I| \lambda^{-2 n}$. Next, we define in the same way the family $B_{n}$. It is formed by the intervals $J$ of this partition of $n$ 'th generation, which are contained in some interval of the family $B_{n-1}$ and for which $\nu(J)<\lambda^{-n}$. Then

$$
\operatorname{Leb}\left(\bigcup\left\{J: J \in B_{n}\right\}\right) \geq\left(1-\frac{1}{\lambda}-\frac{1}{\lambda^{2}}-\cdots-\frac{1}{\lambda^{n}}\right)|I| .
$$

For any $t \in I$ let $J_{n}=J_{n}(t)$ be the interval of the $n$ 'th partition such that $t \in J_{n}$. Thus, for every $t \in \bigcap_{n=1}^{\infty} \bigcup_{J \in B_{n}} J$, we have that $J_{n}(t) \in B_{n}$. Consequently, for all $t \in \bigcap_{n=1}^{\infty} \bigcup_{J \in B_{n}} J$, it holds that $\nu\left(J_{n}(t)\right)<\lambda^{-n}$ for all $n \geq 1$. Let now

$$
C_{n}=\left\{t \in I:\left[t-|I| \lambda^{-3 n}, t+|I| \lambda^{-3 n}\right] \subset J_{n}(t)\right\} .
$$

It is easy to see that $\operatorname{Leb}\left(C_{n}^{c}\right)<2|I| \lambda^{-n}$, and, therefore,

$$
\operatorname{Leb}\left(\bigcap_{n=1}^{\infty} C_{n}\right)>|I|\left(1-2\left(\frac{1}{\lambda}+\frac{1}{\lambda^{2}}+\ldots\right)\right) \text {. }
$$


A NOTE ON WEAK CONVERGENCE OF SINGULAR INTEGRALS IN METRIC SPACES 5

Finally, setting

$$
I^{\prime}:=\left(\bigcap_{n=1}^{\infty} C_{n}\right) \cap\left(\bigcap_{i=1}^{\infty} \bigcup_{J \in B_{i}} J\right)
$$

completes the proof.

Proof of Theorem 1.1. We can assume that $\mu(X) \leq 1$. We define finite Borel measures on the unit interval for all $z \in \operatorname{spt} \mu$ by

$$
\mu_{z}(F)=\mu\{x \in X: d(x, z) \in F\}, F \subset[0,1] .
$$

Let $A_{z}=\cup_{\lambda>2} I_{z}^{\prime}(\lambda)$ where $I_{z}^{\prime}(\lambda)$ are the sets we obtain after we apply Lemma 2.1 to the measures $\mu_{z}$. Then Lemma 2.1 implies that $\mu_{z}\left(A_{z}\right)=\mu_{z}([0,1])$. Let $G_{z}=\left\{r \in(0,1]: r \in A_{z}\right\}$ and

$$
\mathcal{X}_{B}^{\prime}=\left\{\sum_{i=1}^{n} a_{i} \chi_{B\left(z_{i}, r_{i}\right)}: n \in \mathbb{N}, a_{i} \in \mathbb{R}, z_{i} \in \operatorname{spt} \mu, r_{i} \in G_{z_{i}}\right\} .
$$

Then $\mathcal{X}_{B}^{\prime}$ is dense in $L^{2}(\mu)$.

Let $f, g \in \mathcal{X}_{B}^{\prime}$ such that

$$
f=\sum_{i}^{n} a_{i} \chi_{B_{i}} \text { and } g=\sum_{j}^{m} b_{j} \chi_{S_{j}},
$$

where $a_{i}, b_{j} \in \mathbb{R}$ and $B_{i}, S_{j}$ are closed balls. Then for $0<\delta<\varepsilon$,

$$
\int T_{\mu, \varepsilon}^{k} f(x) g(x) d \mu(x)-\int T_{\mu, \delta}^{k} f(x) g(x) d \mu(x)=\sum_{j=1}^{m} \sum_{i=1}^{n} a_{i} b_{j} \int_{S_{j}} \int_{\delta<d(x, y)<\varepsilon} k(x, y) d \mu(y) d \mu(x) .
$$

Furthermore,

$$
\begin{aligned}
& \left|\int_{\substack{S_{j} \\
\delta<d(x, y)<\varepsilon}} \int_{B_{i}} k(x, y) d \mu(y) d \mu(x)\right| \\
& \quad \leq\left|\int_{\substack{B_{i} \cap S_{j} \\
\delta<d(x, y)<\varepsilon}} \int_{B_{i} \cap S_{j}} k(x, y) d \mu(y) d \mu(x)\right|+\left|\int_{\substack{S_{j} \backslash B_{i} \\
\delta<d(x, y)<\varepsilon}} \int_{B_{i} \cap S_{j}} k(x, y) d \mu(y) d \mu(x)\right| \\
& \quad+\left|\int_{\substack{S_{j} \backslash B_{i} \\
\delta<d(x, y)<\varepsilon}} \int_{\substack{B_{i} \backslash S_{j} \\
\delta(x)}} k(x, y) d \mu(y) d \mu(x)\right|+\left|\int_{\substack{S_{j} \cap B_{i} \\
\delta<d(x, y)<\varepsilon}} \int_{B_{i} \backslash S_{j}} k(x, y) d \mu(y) d \mu(x)\right| \\
& \quad \leq \int_{\substack{B_{i} \\
\delta<d(x, y)<\varepsilon}} \int_{\substack{B_{i}^{c} \\
\mid k(x, y)}}\left|k(x) d \mu(y) d \mu(x)+2 \int_{S_{j}} \int_{\substack{S_{j}^{c} \\
\delta<d(x, y)<\varepsilon}}\right| k(x, y) \mid d \mu(y) d \mu(x) .
\end{aligned}
$$


The last inequality follows because by antisymmetry and Fubini's theorem

$$
\int_{\substack{B_{i} \cap S_{j} \\ \delta<d(x, y)<\varepsilon}} \int_{B_{i} \cap S_{j}} k(x, y) d \mu(y) d \mu(x)=0 .
$$

Therefore it is enough to show that for any "good" ball $B=B(z, r)$ with $z \in \operatorname{spt} \mu$ and $r \in G_{z}$

$$
\lim _{\substack{0<\delta<\varepsilon \\ \varepsilon \rightarrow 0}} \int_{\substack{B<d(x, y)<\varepsilon \\ \delta(x)}}|k(x, y)| d \mu(y) d \mu(x)=0,
$$

which will follow by the monotone convergence theorem if we show that

$$
\int_{B} \int_{B^{c}}|k(x, y)| d \mu(y) d \mu(x)<\infty .
$$

Since $B=B(z, r)$ and $r \in G_{z}$ Lemma 2.1 implies that $\mu(\partial B)=0$ hence it is enough to show that

$$
\int_{B^{o}} \int_{B^{c}}|k(x, y)| d \mu(y) d \mu(x)<\infty
$$

where $B^{o}$ stands for the interior of $B$. For any $x \in B^{o}$ let $n(x)>0$ such that

$$
2^{n(x)} d(x, \partial B)=3
$$

and $N(x)=$ integer part of $n(x)+1$. Therefore, since diam $(B) \leq 1$,

$$
B(x, 2) \backslash B \subset \cup_{i=1}^{N(x)} B\left(x, 2^{i} d(x, \partial B)\right) \backslash B\left(x, 2^{i-1} d(x, \partial B)\right) .
$$

Hence for all $x \in B^{o}$

$$
\begin{aligned}
\int_{B(x, 2) \backslash B}|k(x, y)| d \mu(y) & \leq \int_{B(x, 2) \backslash B} d(x, y)^{-s} d \mu(y) \\
& =\sum_{i=1}^{N(x)} \int_{B\left(x, 2^{i} d(x, \partial B) \backslash B\left(x, 2^{i-1} d(x, \partial B)\right)\right.} d(x, y)^{-s} d \mu(y) \\
& \leq \sum_{i=1}^{N(x)} \mu\left(B\left(x, 2^{i} d(x, \partial B)\right)\left(2^{i-1} d(x, \partial B)\right)^{-s} d \mu(y)\right. \\
& \lesssim N(x) \lesssim|\log d(x, \partial B)|,
\end{aligned}
$$

and

$$
\begin{aligned}
\int_{B^{c}}|k(x, y)| d \mu(y) & \lesssim \int_{B(x, 2)^{c}} d(x, y)^{-s} d \mu(y)+|\log d(x, \partial B)| \\
& \lesssim 1+|\log d(x, \partial B)| .
\end{aligned}
$$


Since $r \in G_{z}$ there exists some $\lambda \in \mathbb{N}$ such that $r \in I_{z}^{\prime}(\lambda)$. We write,

$$
\begin{aligned}
\int_{B(z, r)^{o}}|\log d(x, \partial B)| d \mu(x)= & \int_{B\left(z, r-\lambda^{-3}\right)^{o}}|\log d(x, \partial B)| d \mu(x) \\
& +\sum_{n=1}^{\infty} \int_{\left\{x: r-\lambda^{-3 n} \leq d(z, x)<r-\lambda^{-3(n+1)}\right\}}|\log d(x, \partial B)| d \mu(x)
\end{aligned}
$$

Notice that by Lemma 2.1

$$
\begin{gathered}
\mu\left(\left\{x: r-\lambda^{-3 n} \leq d(z, x)<r-\lambda^{-3(n+1)}\right\}\right)=\mu_{z}\left(\left[r-\lambda^{-3 n}, r-\lambda^{-3(n+1)}\right)\right) \\
\leq \mu_{z}\left(\left[r-\lambda^{-3 n}, r+\lambda^{-3 n}\right)\right) \leq \lambda^{-n} .
\end{gathered}
$$

Therefore,

$$
\int_{B(z, r)^{o}}|\log d(x, \partial B)| d \mu(x) \lesssim 3 \log (\lambda)\left(r-\lambda^{-3}\right)^{s}+\sum_{i=1}^{n} \lambda^{-n}\left|\log \left(\lambda^{-3(n+1)}\right)\right|<\infty
$$

and this completes the proof of Theorem 1.1 .

\section{REFERENCES}

[C] V. Chousionis, Singular integrals on Sierpinski gaskets, Publ. Mat. 53 (2009), no. 1, 245256.

[CMPT] V. Chousionis, J. Mateu, L. Prat and X. Tolsa, Calderón-Zygmund kernels and rectifiability in the plane, Adv. Math. 231:1 (2012), 535-568.

$[\mathrm{CM}] \quad$ V. Chousionis and P. Mattila, Singular integrals of general measures separated by Lipschitz graphs, Bull. London Math. Soc. 42 (2010), no. 1, 109-118.

[D] G.David, Des intégrales singulières bornées sur un ensemble de Cantor, C. R. Acad. Sci. Paris Sr. I Math. 332 (2001), no. 5, 391-396.

[DS] G. David and S. Semmes. Singular Integrals and rectifiable sets in $\mathbb{R}^{n}:$ Au-delà des graphes lipschitziens. Astérisque 193, Société Mathématique de France (1991).

[F] H. Federer. Geometric Measure Theory Springer-Verlag, 1969.

$[\mathrm{H}] \quad$ P. Huovinen. A nicely behaved singular integral on a purely unrectifiable set. Proc. Amer. Math. Soc. 129 (2001), no. 11, 3345-3351.

[M] P. Mattila, Geometry of Sets and Measures in Euclidean Spaces, Cambridge University Press, (1995).

[MMV] P. Mattila, M. Melnikov and J. Verdera, The Cauchy integral, analytic capacity, and uniform rectifiability. Ann. of Math. (2) 144 (1996), no. 1, 127-136.

[MV] P. Mattila, J. Verdera, Convergence of singular integrals with general measures, J. Eur. Math. Soc. (JEMS) 11 (2009), no. 2, 257-271.

[MP] P. Mattila, D. Preiss, Rectifiable measures in $\mathbb{R}^{n}$ and existence of principal values for singular integrals, J. London Math. Soc., 52 (1995), 482-496.

$[\mathrm{NToV}]$ F. Nazarov, X. Tolsa and A. Volberg, On the uniform rectifiability of AD-regular measures with bounded Riesz transform operator: the case of codimension 1. submitted (2012).

[T] X.Tolsa, Principal values for Riesz transforms and rectifiability, J. Funct. Anal. 254 (2008), no. 7, 1811-1863.

[SUZ] M. Szostakiewicz, M. Urbański, and A. Zdunik, Fine Inducing and Equilibrium Measures for Rational Functions of the Riemann Sphere, Preprint 2011. 
Department of Mathematics, University of Illinois, 1409 West Green St., UrBANA, IL 61801

E-mail address: vchous@math.uiuc.edu

Department of Mathematics, University of North Texas, General Academics Building 435, 1155 Union Circle \#311430, Denton, TX 76203-5017

E-mail address: urbanski@unt.edu 